\title{
Field Trial of WDM-OTDM Transmultiplexing employing Photonic Switch Fabric-based Buffer-less Bit-interleaved Data Grooming and All-Optical Regeneration
}

\author{
G. Zarris ${ }^{1}$, F. Parmigiani ${ }^{2}$, E. Hugues-Salas ${ }^{1}$, R. Weerasuriya ${ }^{3}$, D. Hillerkuss ${ }^{4}$, N. Amaya \\ Gonzalez $^{1}$, M. Spyropoulou ${ }^{5}$, P. Vorreau ${ }^{4}$, R. Morais ${ }^{6}$, S.K. Ibrahim ${ }^{3}$, D. Klonidis ${ }^{5}$, P. \\ Petropoulos $^{2}$, A.D. Ellis ${ }^{3}$, P. Monteiro ${ }^{6}$, A. Tzanakaki ${ }^{5}$, D. Richardson ${ }^{2}$, I. Tomkos ${ }^{5}$, R. \\ Bonk $^{4}$, W. Freude ${ }^{4}$, J. Leuthold ${ }^{4}$, and D. Simeonidou ${ }^{1}$ \\ 1 - Photonic Networks Laboratory, University of Essex, Colchester CO4 3SQ, U.K., Email: gzarris@essex.ac.uk \\ 2 - Optoelectronics Research Centre, University of Southampton, U.K., 3 - Photonic Systems Group, Department of \\ Physics and Tyndall National Institute, University College Cork, Ireland, 4 - Institute of Photonics and Quantum \\ Electronics, University of Karlsruhe, 76131 Karlsruhe, Germany 5 - Athens Information Technology Centre, Athens, \\ Greece, 6 - Nokia Siemens Networks Portugal S.A., 2720 Amadora, Portugal
}

\begin{abstract}
We report, for the first time, a field trial of a novel $42.7 \mathrm{Gbps} / 128.1 \mathrm{Gbps}$ WDM/OTDM grooming node, and confirm node interoperability and the data integrity of asynchronous retiming. (C)2009 Optical Society of America

OCIS codes: (060.4230) Multiplexing, (060.1155) All-optical networks
\end{abstract}

\section{Introduction}

The rapid growth in network traffic rates will increase the significance of data grooming for two reasons: First, its share in the overall network resources is set to increase; and secondly, current, predominantly electronic, methods of traffic grooming make for one of the least scalable aspects of today's technology in terms of cost and power consumption [1]. As a result, innovative solutions to address data grooming are being sought [2], based on a host of optical and electronic technologies, and different paradigms of multi-granularity. In future high capacity networks a coarse granularity of 100 's Gb/s may be desirable. In this context, optical time division multiplexing (OTDM) may find increasing relevance. Nevertheless, to realise the potential benefits of OTDM in a multi-granular network, techniques and technologies for the efficient access and switching of tributaries need to be developed.

In this paper we present, for the first time, the results of two field experiments involving a WDM-OTDM grooming switch capable of providing time-slot, wavelength, and space switching [3]. The technologies used are highly scalable in terms of bit-rates and offer the potential of very low power consumption. The switch could be applicable to edge/core and core traffic grooming in the future network.

\section{Optical grooming switch architecture}

Fig. 1a depicts the grooming switch architecture [3], which interconnects up to three $43 \mathrm{~Gb} / \mathrm{s}$ channels - selected using MEMS switch from lower bit-rate WDM domains - with an OTDM domain which carries $129 \mathrm{~Gb} / \mathrm{s}$ (3x43G) bit-interleaved OTDM channels. It consists of three main building blocks, namely, WDM-OTDM with time-slot interchange (TSI), OTDM-WDM demultiplexing, and all-optical 2R regeneration. All $43 \mathrm{~Gb} / \mathrm{s}$ channels, whether originating in the OTDM domain or the WDM domain, are input in a MEMS space switch, which provides nonblocking circuit connectivity. In more detail, first, the WDM-OTDM consists of three dual-gate asynchronous digital optical regenerator (ADORE) units [4], each mapping one $43 \mathrm{~Gb} / \mathrm{s}$ on/off keying (OOK) channel on one OTDM time-slot. TSI can be performed by altering the mapping of WDM channels onto ADORE units.

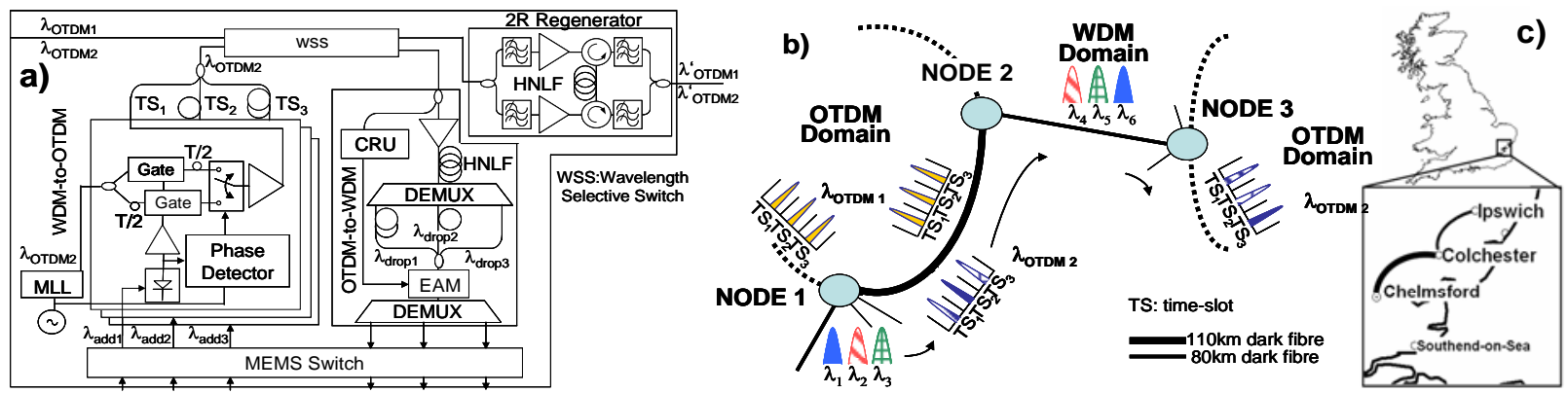

Fig. 1a. Grooming switch architecture [3]; 1b. Network scenario; 1c. Dark fibre network 
The ADORE provides regeneration, retiming, pulse width adaptation and wavelength conversion onto a local modelocked laser (MLL). The retiming action, in particular, concerns the translation of the input signal frequency to a node-specific frequency determined by the node internal clock. In this way, and without the need for electronic buffers, all WDM tributaries end up with precisely the same clock frequency, which is necessary for bit-interleaving tributaries to form an OTDM channel. Retiming action, nevertheless, means that bit-slot slippages take place. It is therefore necessary to provide a mechanism for maintaining data integrity in grooming (see section 3). Second, OTDM-WDM is achieved by wavelength conversion using offset filtering of self-phase modulation (SPM) broadened spectrum in highly non-linear fibre (HNLF), followed by a single electro-absorption modulator (EAM) based optical gate [5]. A clock recovery unit (CRU) [6] is used here to provide the EAM with a synchronous clock signal. Third, the $2 \mathrm{R}$ regenerator is also based on offset filtering of SPM broadened spectrum in HNLF, and can simultaneously process two $129 \mathrm{Gbit} / \mathrm{s}$ wavelengths, if bidirectional architecture is considered [7].

\section{Field experiments and results}

The field trial was performed using two dispersion compensated SMF- $28^{\mathrm{TM}}$ dark fibre sections (Fig. 1c). The first section, Colchester-Ipswich, was $100 \%$ pre compensated using slope matched dispersion compensating module and had a round trip length of $80 \mathrm{~km}$ and represented an access link transmitting $42.7 \mathrm{~Gb} / \mathrm{s}$ channels. The second, Colchester-Chelmsford represented a link in the core network, had a round trip length of $110 \mathrm{~km}$ and was $80 \%$ pre compensated and $20 \%$ post compensated and carried $129 \mathrm{~Gb} / \mathrm{s}$ channels.

Two experiments were performed with the aim of demonstrating key network functions in the following scenario (Fig. 1b). Node 1 performs WDM-OTDM grooming of traffic which originates in an edge $43 \mathrm{~Gb} / \mathrm{s} \mathrm{WDM}$ domain. In Node 2 a single 2R regenerator regenerates two $129 \mathrm{~Gb} / \mathrm{s}$ channels. The originally groomed OTDM channel is subsequently OTDM-WDM demultiplexed. Node 3 retimes one of these WDM tributaries and, together with other two local $43 \mathrm{~Gb} / \mathrm{s}$ channels, it forms a new OTDM channel which is launched in the second OTDM domain. The above network scenario is demonstrated in two separate experiments, with the first experiment demonstrating the interoperability of Nodes $1 \& 2$, and the second the interoperability of Nodes $2 \& 3$.
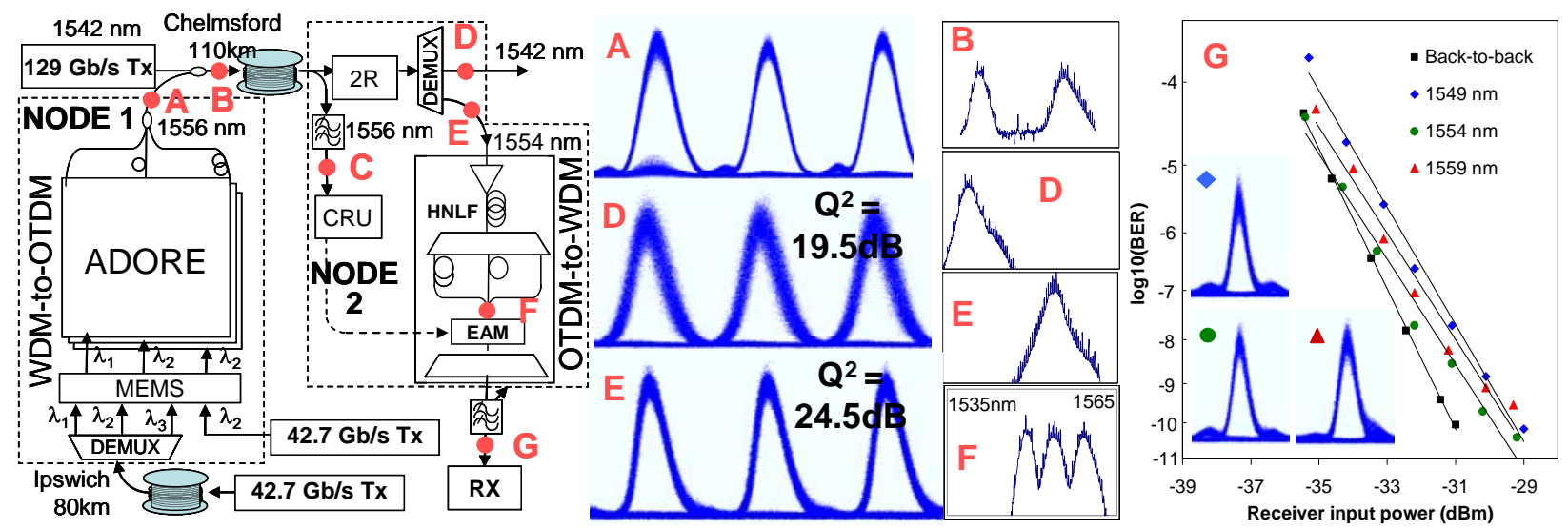

Fig. 2. Experiment 1 setup and results - OTDM grooming, transmission, and tributary separation.

In the first experiment (Fig. 2), $3 \times 43 \mathrm{~Gb} / \mathrm{s} 33 \%$ RZ OOK channels at wavelengths $\lambda_{1}=1547.7 \mathrm{~nm}, \lambda_{2}=1549.3 \mathrm{~nm}$ and $\lambda_{3}=1550.9 \mathrm{~nm}$ were transmitted in Ipswich link. The data pattern consisted of a repeating $2^{7}-1$ pseudo random bit sequence (PRBS) of $1 \mathrm{~ms}$ duration, and a single modified $2^{19}-1$ PRBS serving as a $1 \mu$ s guard-band with mark ratio $52.5 \%$. Of the three transmitted channels, $\lambda_{1}$ subsequently entered an ADORE unit, which was configured as in $[4,8]$, including a $15 \mathrm{~dB}$ gain pre-amplifier with no optical band-pass filter. The ADORE detected the guard-band by simple power measurement, and a control circuit allowed selection of the correct phase for each $1 \mathrm{~ms}$ burst (i.e. allowing for bit-slot slippages) with a 440ns switching time. In this way, data block integrity was assured during natural variations of input data phase in the dark fibre. For certain tests we used a computer controlled tunable delay at the ADORE input to accelerate the relative variation of input phase up to 9ps per second max. The left eye in inset A, Fig. 2 shows the ADORE output eye over a controlled 1 bit (i.e. 23ps) sweep, at step size of $0.1 \mathrm{ps}$, of the input phase. During such controlled phase sweeps we recorded, in an error detector (ED) gated at the edges of the guardband, error free operation (zero errors) down to $-27 \mathrm{dBm}$ RX power, suggesting an approximate penalty of $3-4 \mathrm{~dB}$ due to eye closure, which is in agreement with previous stand-alone testing [8]. Two local channels at $\lambda_{2}$ were pulsewidth adapted, wavelength converted onto the same MLL, and interleaved together with the ADORE output to form 
the OTDM channel at $1556 \mathrm{~nm}$ (inset A, Fig. 2). A second $129 \mathrm{~Gb} / \mathrm{s}$ OTDM channel at $1542 \mathrm{~nm}$ was generated using a semiconductor-based MLL and was transiting the node. After transmission, both OTDM channels were $2 \mathrm{R}$ regenerated [7], applying $2 \mathrm{~nm}$ blue-offset filtering. The $1542 \mathrm{~nm}$ channel was restored to its pre-transmission quality, according to $\mathrm{Q}^{2}$ and BER measurements. The regenerated $1556 \mathrm{~nm}$ was exhibiting an open eye of $\mathrm{Q}^{2} 24.5 \mathrm{~dB}$, irrespectively of the input data phase to the ADORE. This was subsequently OTDM-WDM demultiplexed into wavelengths 1549, 1554 and 1559nm, and burst mode BER measurements are shown in Fig. 2, along with the backto-back EAM-demultiplexed $1556 \mathrm{~nm}$ OTDM channel. The maximum penalty is $2 \mathrm{~dB}$. The degradation was mainly due to the presence of a small leading pulse from the MLL source that was spreading during transmission into adjacent pulses causing beating, thus affecting CRU performance.

In the second experiment (Fig. 3), an improved MLL source was used, along with improved filtering in the OTDM-WDM sub-system. A $129 \mathrm{~Gb} / \mathrm{s}$ OTDM at $1556 \mathrm{~nm}$ was formed by interleaving three $43 \mathrm{~Gb} / \mathrm{s}$ tributaries of the same data pattern (incl. guard-bands) as in the first experiment. The OTDM channel was launched in the Chelmsford link and was subsequently $2 \mathrm{R}$ regenerated with $2 \mathrm{~nm}$ blue-offset filtering. The regenerated signal was then OTDM-WDM demultiplexed to wavelengths $\lambda_{4}=1549, \lambda_{5}=1554$, and $\lambda_{6}=1559 \mathrm{~nm}$. All three demuxed channels were transmitted in the Ipswich link. At this point, BER measurements show no penalty on the 1554 and $1559 \mathrm{~nm}$ tributaries, compared with the back-to-back EAM-demuxed $1556 \mathrm{~nm}$ channel. However, the $1549 \mathrm{~nm}$ shows a small, $0.5 \mathrm{~dB}$ penalty, and an error floor (below $10^{-12}$ ). This is due to insufficient broadening in the OTDM-WDM HNLF in the shorter wavelength side. Next, the $1559 \mathrm{~nm}$ channel was input to one ADORE. The ADORE output eye was confirmed to be error free (zero errors) for a whole 1 bit period (23ps) sweep of the input data phase. Along with two other tributaries generated from two local $42.7 \mathrm{~Gb} / \mathrm{s} 33 \%$ RZ channels, this tributary was then groomed on a 129 $\mathrm{Gb} / \mathrm{s}$ OTDM channel at $1556 \mathrm{~nm}$. Once again, the eye diagram confirms that the $2 \mathrm{R}$ regenerator is capable of restoring the ADORE eye for transmission into the new OTDM domain.
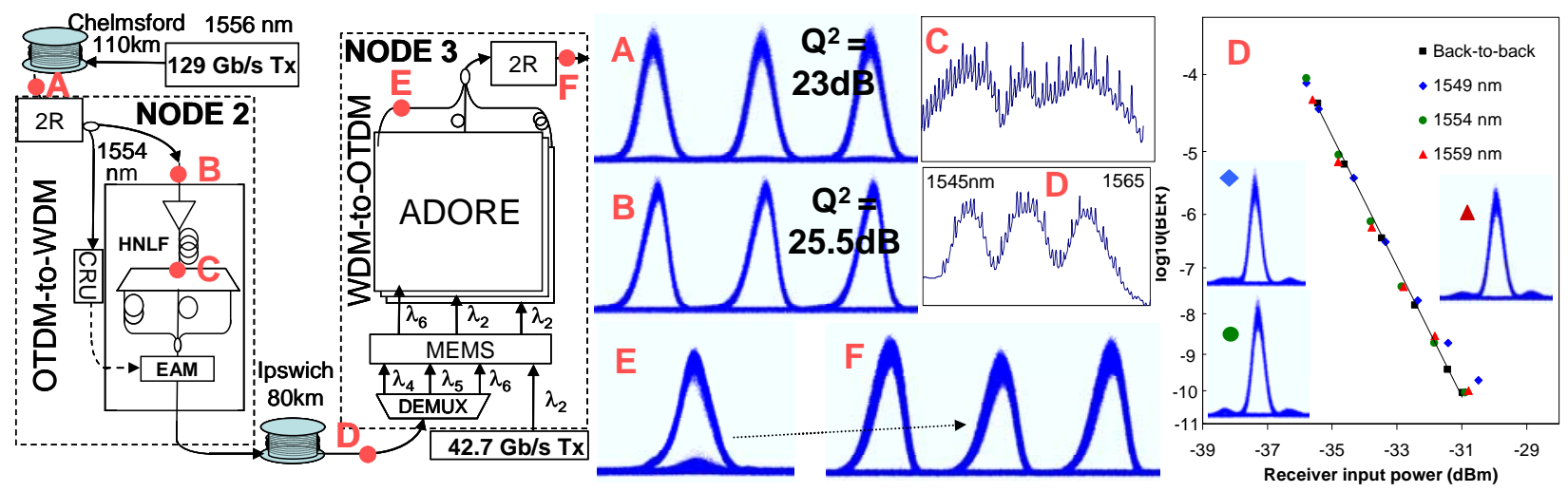

Fig. 3 Experiment 2 setup and results - OTDM transmission, tributary separation, WDM bridge and re-aggregation

\section{Conclusions}

Our results confirm, first, the data integrity of the asynchronous retiming scheme in both cases of edge traffic grooming and OTDM domain interconnection and, second, the potential of the all-optical techniques used for signal regeneration and wavelength conversion in small scale OTDM domains. The great potential of the asynchronous retiming scheme in transparently interconnecting OTDM domains will be discussed at the conference.

This work was supported by the European project TRIUMPH (grant IST-027638 STP), JANET UK, and the Science Foundation Ireland (grant 06/IN/I969). The authors acknowledge equipment loan from Agilent Technologies, U.K.

\section{References}

[1] A.A.M. Saleh et al., "Evolution towards the next-generation core optical network", IEEE JLT, vol.24, no.9, p.3303-3321 (2006)

[2] R. Nejabati, et al., "Multigranular Optical Router for Future Networks", OSA J. of Optical Networking, vol.7, issue 11, p.914-927 (2008)

[3] P. Vorreau, et al, "2R/3R optical grooming switch with time-slot interchange", Proc. ECOC 2008, PDP Th.3.F.4 (2008)

[4] S.K. Ibrahim, et al,"Novel 42.65Gbit/s dual gate asynchronous digital optical regenerator using a single MZM", Proc. ECOC, Tu.4.D.3 (2008)

[5] R. Morais et al., "OTDM-to-WDM Conversion based on Wavelength Conversion and Time Gating in a Single Optical Gate", Proc. OFC, OTuD5 (2008)

[6] J. Lasri, et al., "Ultralow timing jitter 40-gb/s clock recovery using a self-starting optoelectronic oscillator," IEEE PTL, vol. 16, no. 1, p. 263265 (2004)

[7] F. Parmigiani, et al., "2R regeneration of two $130 \mathrm{Gbit} / \mathrm{s}$ channels within a single fiber", Proc OFC, JThA56 (2009)

[8] G. Zarris et al., "WDM-to-OTDM Traffic Grooming by means of Asynchronous Retiming", Proc. OFC, OThJ6 (2009) 